\title{
Decoding of Methylated Histone H3 Tail by the Pygo-BCL9 Wnt Signaling Complex
}

\author{
Marc Fiedler, ${ }^{1,3}$ María José Sánchez-Barrena, ${ }^{1,3}$ Maxim Nekrasov, ${ }^{2}$ Juliusz Mieszczanek, ${ }^{1}$ Vladimir Rybin, ${ }^{2}$ Jürg Müller, ${ }^{2}$ \\ Phil Evans, ${ }^{1}$ and Mariann Bienz ${ }^{1, *}$ \\ ${ }^{1} \mathrm{MRC}$ Laboratory of Molecular Biology, Hills Road, Cambridge CB2 OQH, UK \\ 2EMBL, Meyerhofstrasse 1, 69117 Heidelberg, Germany \\ ${ }^{3}$ These authors contributed equally to this work as first authors. \\ ${ }^{*}$ Correspondence: mb2@mrc-Imb.cam.ac.uk \\ DOI 10.1016/j.molcel.2008.03.011
}

\section{SUMMARY}

Pygo and BCL9/Legless transduce the Wnt signal by promoting the transcriptional activity of $\beta$-catenin/ Armadillo in normal and malignant cells. We show that human and Drosophila Pygo PHD fingers associate with their cognate HD1 domains from BCL9/Legless to bind specifically to the histone $\mathrm{H} 3$ tail methylated at lysine 4 (H3K4me). The crystal structures of ternary complexes between PHD, HD1, and two different $\mathrm{H} 3 \mathrm{~K} 4 \mathrm{me}$ peptides reveal a unique mode of histone tail recognition: efficient histone binding requires HD1 association, and the PHD-HD1 complex binds preferentially to $\mathrm{H} 3 \mathrm{~K} 4 \mathrm{me} 2$ while displaying insensitivity to methylation of H3R2. Therefore, this is a prime example of histone tail binding by a PHD finger (of Pygo) being modulated by a cofactor (BCL9/ Legless). Rescue experiments in Drosophila indicate that Wnt signaling outputs depend on histone decoding. The specificity of this process provided by the Pygo-BCL9/Legless complex suggests that this complex facilitates an early step in the transition from gene silence to Wnt-induced transcription.

\section{INTRODUCTION}

The canonical Wnt signaling pathway controls numerous transcriptional switches during the normal development of animals, and also the homeostatic self-renewal of adult tissues (Clevers, 2006). Inappropriate activation of this pathway often leads to cancer, especially in the intestinal epithelium (Bienz and Clevers, 2000; Polakis, 2000). The Wnt-induced transcriptional switches during normal and malignant development are operated by $\beta$-catenin, or Drosophila Armadillo: this key effector of the pathway is activated and stabilized in response to Wnt signaling, and thus binds to TCF/LEF DNA-binding proteins to coactivate the transcription of Wnt target genes (Arce et al., 2006). This process depends on the recruitment of a wide range of different transcriptional coregulators, including chromatin modifier and remodeling complexes, to the $C$ terminus of $\beta$-catenin associated with TCF at Wnt target genes (Willert and Jones, 2006). Notably, these include a SET1-type methyltransferase complex that promotes the trimethylation of lysine 4 in the tail of histone H3 (H3K4) (Sierra et al., 2006).

Pygopus (Pygo) and Legless (Lgs) were discovered in Drosophila as new Wnt signaling components that are essential for Armadillo-mediated transcription during normal development (Belenkaya et al., 2002; Kramps et al., 2002; Parker et al., 2002; Thompson et al., 2002). Similarly, their mammalian orthologs (Pygo1 and Pygo2, and BCL9 and BCL9-2/B9L, respectively) contribute to efficient $\beta$-catenin-mediated transcription in Wntstimulated mammalian cells, and in colorectal cancer cell lines with elevated Wnt pathway activity (Adachi et al., 2004; Brembeck et al., 2004; Thompson et al., 2002). Likewise, during vertebrate development, Pygo proteins modulate $\mathrm{Wnt} / \beta$-catenin responses in multiple tissues (Lake and Kao, 2003; Li et al., 2007a; Schwab et al., 2007; Song et al., 2007).

Molecularly, BCL9/Lgs proteins function as adaptors between Pygo and Armadillo/ $\beta$-catenin, by binding through their homology domain 1 (HD1) to the PHD finger in the $\mathrm{C}$ terminus of Pygo, and through their homology domain 2 (HD2) to the Armadillo repeat domain of Armadillo/ $\beta$-catenin (Kramps et al., 2002; Städeli and Basler, 2005). These interactions are essential for Wnt responses during normal Drosophila development (Hoffmans and Basler, 2004; Townsley et al., 2004b). It was proposed that the PygoBCL9 complex recruits an unknown transcriptional cofactor to Armadillo/ $\beta$-catenin to support Wnt-induced transcription (Hoffmans et al., 2005; Kramps et al., 2002; Thompson, 2004). Alternatively, this complex may facilitate efficient targeting of Armadillo/ $\beta$-catenin to TCF target genes with which Pygo is associated even in the absence of Wnt signaling (de la Roche and Bienz, 2007; Townsley et al., 2004a), possibly reflecting an ability of its PHD finger to associate with chromatin (Bienz, 2006). Indeed, it was discovered recently that several PHD fingers can bind with high specificity and selectivity to trimethylated lysine 4 of histone $\mathrm{H} 3$ (H3K4me3), namely BPTF (Li et al., 2006; Wysocka et al., 2006), ING2/Yng1 (Pena et al., 2006; Shi et al., 2006, 2007; Taverna et al., 2006) and RAG2 (Ramon-Maiques et al., 2007). By contrast, the PHD finger of $\mathrm{BHC} 80$ binds to unmethylated histone $\mathrm{H} 3$ tail (H3K4me0) (Lan et al., 2007). Different types of PHD fingers thus act to read the histone code (Jenuwein and Allis, 2001; Kouzarides, 2007), and in particular the methylation status of specific histone tails (Corsini and Sattler, 2007; Taverna et al., 2007).

Here, we report the crystal structure of the human PHD-HD1 complex. We show that complex formation is critical for highaffinity binding of human and Drosophila Pygo PHD fingers to $\mathrm{H} 3 \mathrm{~K} 4 \mathrm{me}$, and that the binding of Pygo to methylated histone 
A

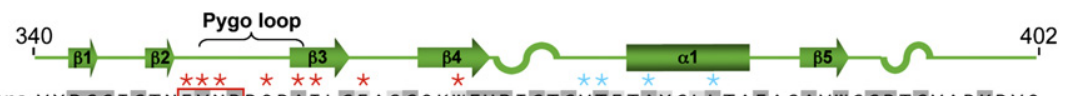

H. sapiens VYPCGICTNEVNDDQDAILCEASCQKWFHRICTGMTETAYGLLTAEASAVWGCDTCMADKDVQ

D. rerio VFPCGLCMSEVHDDQEAILCEASCQRWFHRDCTGLTEPAYGLLTRESAAVWACDFCLKTKEIQ

S. purpuratus LFPCGICHQQVQDSEDAVICVSSCHTWFHRICTGMTTTAYTLLNSEHAAEWVCDRCVREKKIP

D. melanogaster IYPCGMC $\underbrace{H K E V N D N D E A V F C E S G C N F F F H R T C V G L T E A A F Q M L N K E V F A E W C C D K C V S S K H I P}_{\text {loop1 }}$

B

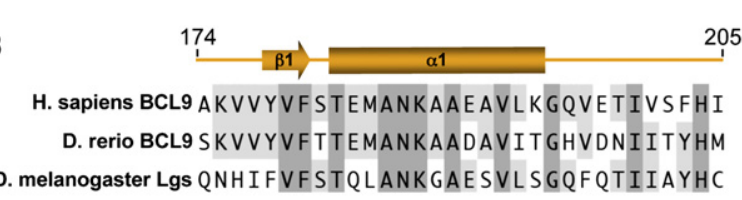

205

$c \stackrel{n}{\text { end }}$

D

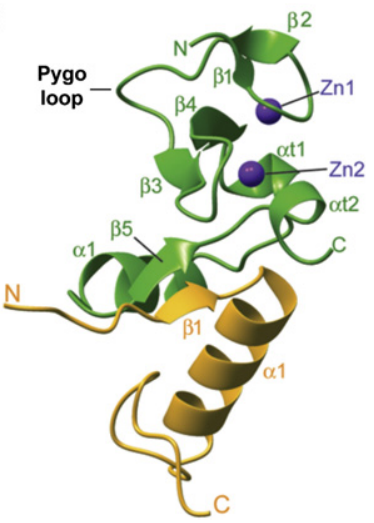

E

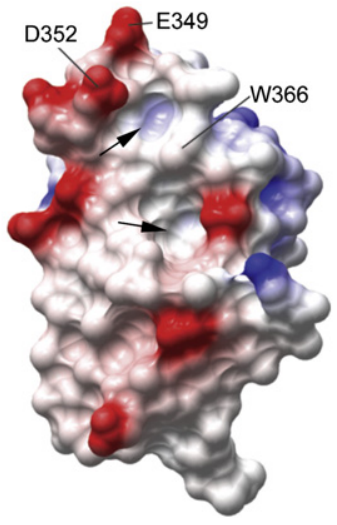

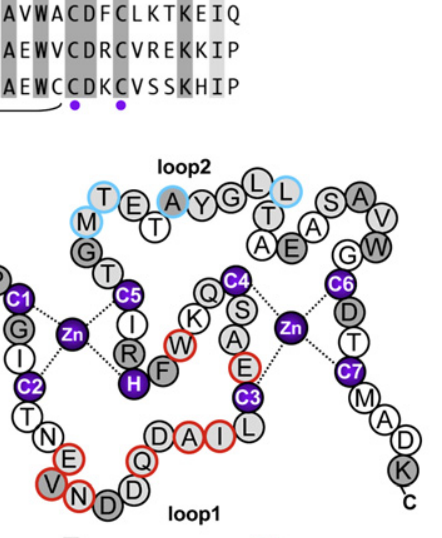

2

(1)

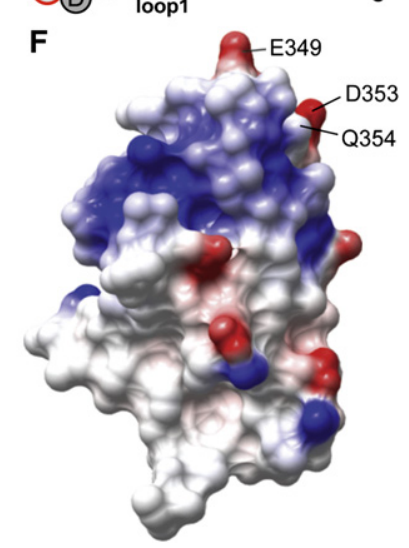

Figure 1. Sequence Alignments and Structure of the Human PHD-HD1 Complex

(A and B) Alignments of (A) PHD sequences of hPygo1 (Q9Y3Y4), zebrafish Pygo2 (Q1L8T6), sea urchin Pygo (XP_791313), and Drosophila Pygo (Q9V9W8), and (B) HD1 sequences of hBCL9 (O00512), zebrafish BCL9 (Q67FY0), and Drosophila Lgs (Q961D9). Dark gray, invariant residues; light gray, semiconserved residues. Marked above sequences are secondary structure elements ( $\beta$ sheets, $\alpha$ helices; $\alpha$ turns are marked by $S$ shapes) and residues involved in HD1 (blue) or H3K4me binding (red) as defined by mutational analysis (Figures 3B and 4E). EVND motif is boxed; indicated are also $\mathrm{Zn}^{2+}$-coordinating residues (purple), Pygo loop, and loop1 and loop2 surfaces (brackets).

(C) Crossbrace ligation of hPygo1 PHD finger, with Zn-coordinating and mutated residues highlighted (colors as in [A]).

(D) Ribbon representation of the hPHD-HD1 complex structure solved at $1.59 \AA$ resolution, with secondary structure elements labeled as in (A) and (B). PHD, green; HD1, orange; $\mathrm{Zn}^{2+}$, purple.

(E and F) Molecular surface representations of hPHD-HD1, colored according to electrostatic potential (red, negative charges; blue, positive charges), with some Pygo loop and EVND residues labeled. (E) View similar to (D), showing the PHD loop1 surface, with two conspicuous cavities (arrows) separated by W366; (F) is rotated $180^{\circ}$ with respect to $(\mathrm{E})$.

H3 tail is physiologically relevant during Drosophila development. The structure of two ternary complexes between PHD, HD1, and differently methylated histone $\mathrm{H} 3$ peptides reveals that the Pygo1 PHD finger has two distinct surfaces that are engaged simultaneously in binding to HD1 and H3K4me. Our work pinpoints three unique features of this finger: preference for $\mathrm{H} 3 \mathrm{~K} 4 \mathrm{me}$, insensitivity to methylation of arginine 2 (H3R2), and assistance by HD1 in its recognition of the methylated histone $\mathrm{H} 3$ tail.

\section{RESULTS}

To understand how Pygo proteins interact with BCL9, we coexpressed the PHD finger from hPygo1 with its cognate HD1 domain from human BCL9 (Figures 1A and 1B) in bacteria, and purified the resulting complex (hPHD-HD1, the "binary complex"), which was stable over gel filtration and exhibited equimolar binding (see Figure S1 available online). It produced diffracting crystals in several different crystallization conditions, which enabled us to determine its structure by X-ray crystallography at $1.59 \AA$ resolution (Tables 1 and 2).

\section{The Structure of the Human PHD-HD1 Complex}

The overall fold of the Pygo PHD finger, like that of other PHD fingers (Bienz, 2006), forms a compact scaffold that binds two $\mathrm{Zn}^{2+}$ ions in a crossbraced fashion (Figures $1 \mathrm{~A}$ and $1 \mathrm{C}$ ), as previously shown (Nakamura et al., 2007). It consists of two pairs of antiparallel $\beta$ strands (whereby $\beta 3-\beta 4$ is a secondary structural element seen in all known PHD fingers), followed by an $\alpha$ helix and another $\beta$ strand 


\section{Molecular Cell}

H3 Tail Recognition by the Pygo-BCL9 Complex

\begin{tabular}{|c|c|c|c|c|}
\hline hPHD_W366F-HD1 & Peak (P) & Inflexion (Ip) & & Remote (Rm) \\
\hline Beamline & ESRF, ID23-1 & & & \\
\hline Strategy & $180^{\circ}, \Delta \varphi 1^{\circ}$ & $180^{\circ}, \Delta \varphi 1^{\circ}$ & & $180^{\circ}, \Delta \varphi 1^{\circ}$ \\
\hline Wavelength & 1.2832 & 1.2841 & & 1.0000 \\
\hline Space Group & 1222 & & & \\
\hline$a, b, c(\AA)$ & $32.56,71.91,91.56$ & & & \\
\hline$\alpha, \beta, \gamma\left({ }^{\circ}\right)$ & $90.0,90.0,90.0$ & & & \\
\hline Resolution $(\AA ̊)$ & $30.67-1.96(2.07-1.96)^{\mathrm{a}}$ & $35.97-1.96(2.07-1.96)$ & & $24.96-1.65(1.74-1.65)$ \\
\hline $\mathrm{R}_{\text {merge }}(\%)^{\mathrm{b}}$ & $4.6(11.6)$ & $4.7(13.8)$ & & $5.3(38.0)$ \\
\hline $\mathrm{l} / \sigma(\mathrm{l})$ & $30.7(13.8)$ & $29.1(11.5)$ & & $21.7(4.7)$ \\
\hline Completeness (\%) & 99.9 (99.9) & $99.9(99.9)$ & & $99.6(99.6)$ \\
\hline Multiplicity & $6.8(6.7)$ & $6.8(6.7)$ & & $6.8(7.0)$ \\
\hline Anomalous Completeness (\%) & $99.8(99.6)$ & $99.8(99.5)$ & & $99.5(100)$ \\
\hline Anomalous Multiplicity & $3.6(3.5)$ & $3.6(3.5)$ & & $3.6(3.6)$ \\
\hline Complexes in A.U. & & & & 1 \\
\hline Phasing & N Acentric (A) & FOM Acentric & N Centric (C) & FOM centric \\
\hline Overall & 11,649 & 0.57 & 1707 & 0.46 \\
\hline Phasing power & Isomorphous (A) & Isomorphous (C) & Anomalous (A) & \\
\hline $\mathrm{Pk}$ & 0.0 & 0.0 & 3.4 & \\
\hline Ip & 2.5 & 1.7 & 2.2 & \\
\hline $\mathrm{Rm}$ & 1.7 & 1.1 & 1.4 & \\
\hline \multicolumn{5}{|l|}{ Refinement } \\
\hline Resolution $(\AA)$ & & & & $24.96-1.65(1.69-1.65)$ \\
\hline Number of reflections & & & & 12,608 \\
\hline Test set size (\%) & & & & 5.0 \\
\hline $\mathrm{R}_{\text {work }}(\%)$ & & & & $21.7(25.8)$ \\
\hline $\mathrm{R}_{\text {free }}(\%)$ & & & & $26.7(29.0)$ \\
\hline Number of atoms (non-H) & & & & 885 \\
\hline Residues (PHD/HD1) & & & & $333-397 / 174-205$ \\
\hline$<B>\left(\AA^{2}\right)$ & & & & 28.7 \\
\hline \multicolumn{5}{|l|}{ Rmsd } \\
\hline Bond length $(\AA)$ & & & & 0.012 \\
\hline Bond angle $\left({ }^{\circ}\right)$ & & & & 1.372 \\
\hline \multicolumn{5}{|l|}{ Ramachandran plot } \\
\hline In favored regions (\%) & & & & 98.0 \\
\hline In allowed regions (\%) & & & & 2.0 \\
\hline Outliers (\%) & & & & 0.0 \\
\hline
\end{tabular}

( $\beta 5)$ in the "loop2" segment (which is unusually large in Pygo proteins; Bienz, 2006), flanked by two $\alpha$ turns (Figures 1A, 1C, and 1D). Note that the "loop1" surface of hPHD contains the highly conserved EVND motif (where V and D are invariant in all known Pygo proteins), which is part of a prominent and flexible surface loop (to be called "Pygo loop," V350-A356; Figures 1A and 1D).

The HD1 domain of BCL9 is a flat module with a secondary structure that comprises an $\mathrm{N}$-terminal tail, which acquires an extended conformation due to contacts with a symmetry-related molecule, followed by a $\beta$ strand, an $\alpha$ helix, and an unstructured $\mathrm{C}$-terminal end that folds back toward the $\mathrm{N}$ terminus (Figures $1 \mathrm{~B}$ and 1D).
The binary complex shows a segregation of charges on its molecular surface, with one face being predominantly negatively (Figure 1E), and the opposite positively, charged (Figure 1F). The former presents two conspicuous hydrophobic cavities (Figure 1E, arrows) surrounded by acidic residues and separated by W366, similar to the histone-binding pockets seen in other PHD fingers.

\section{The Pygo-BCL9 Interface Involves Highly Conserved PHD Loop2 Residues}

Two sets of contacts mediate the interaction between $\mathrm{hPHD}$ and HD1 (Figure 1D). The first involves primarily $\beta$ sheet $\mathrm{H}$ bonds between PHD $\beta 5$ (S387, V389, and G391) and HD1 $\beta 1$ (Y178, V179, 
A

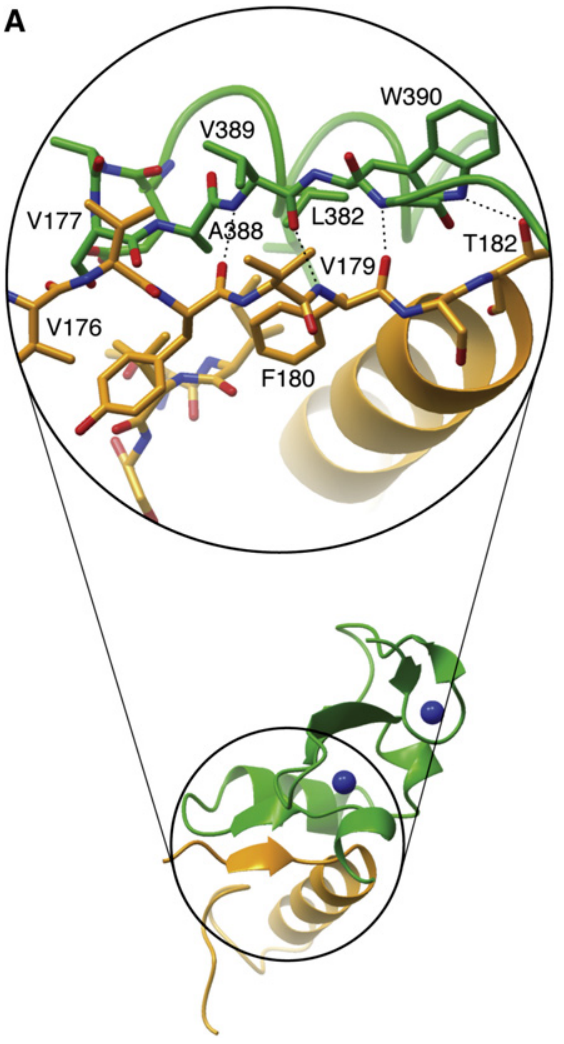

B

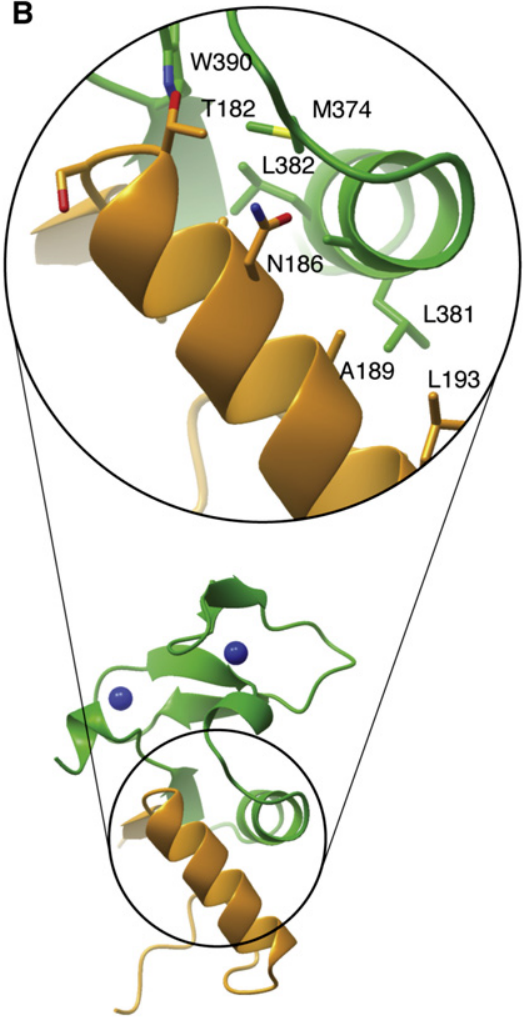

Figure 2. The Pygo-BCL9 Interface Contacts between PHD (green) and HD1 (orange). (A) Parallel $\beta$ sheets (PHD $\beta 5$, HD1 $\beta 1$ ), (B) $\alpha$ helices. $\mathrm{H}$ bonds are indicated by dotted lines; amino acids involved in intermolecular interactions are depicted in cylinder mode.

Pygo) can bind to the histone $\mathrm{H} 3$ tail, and that this binding is mediated by their PHD fingers (data not shown; note that the PHD finger is the only domain identifiable in Pygo proteins by BLAST searches). To test whether Pygo PHD fingers exhibit a preference for H3K4me3, like other PHD fingers (see the Introduction), we chose the more quantitative method of isothermal titration calorimetry (ITC) to determine the affinity of bacterially expressed Pygo PHD fingers for various methylated and unmodified 15-mer histone tail peptides.

Indeed, the PHD finger from hPygo1 binds to H3K4me3 and H3K4me2 with similar high affinities $\left(K_{d}, 2.5\right.$ and 2.4 $\mu \mathrm{M}$, respectively), and to $\mathrm{H} 3 \mathrm{~K} 4 \mathrm{me} 1$ with somewhat reduced affinity $\left(\mathrm{K}_{\mathrm{d}}, 9 \mu \mathrm{M}\right)$. By contrast, its binding to $\mathrm{H} 3 \mathrm{~K} 4 \mathrm{meO}$

and F180) (Figure 2A). These intermolecular interactions depend mainly on backbone atoms. Consistent with this, there is little sequence conservation in this PHD segment, except for A388 (which forms a hydrophobic side-chain interaction with HD1) and W390 (which forms a side-chain interaction with HD1 T182; this tryptophan is also a defining feature of PHD fingers and contributes to their structural cores [Bienz, 2006]).

The second set of contacts comprises a network of hydrophobic side chains, as well as two H bonds (formed by HD1 N186 side chains and PHD backbone atoms; Figure 2B). These side-chain contacts involve either invariant residues (T375, A378, and L382) or a semiconserved PHD residue (M374) (Figure 1A). These were previously mutated to alanine or valine in $\mathrm{APHD}$, and each individual mutation essentially eliminated Lgs binding; importantly, their combined mutation results in complete inactivation of Pygo, as judged by rescue assays in pygo null mutant Drosophila embryos (Townsley et al., 2004b). T375A and L382A mutations also block the HD1 binding of mouse Pygo1 (Townsley et al., 2004b). Likewise, the HD1 residues involved in these hydrophobic interactions are either invariant (T182, A185, N186, A189, and I200) or semiconserved (V201; Figure 1B). Systematic N- and C-terminal deletions of HD1 identified V177-I205 as the minimal PHD-interacting fragment (Figure S2), spanning all of these conserved HD1 residues involved in side-chain contacts with PHD.

\section{Binding of PHD-HD1 Complexes}

to Methylated Histone H3 Tail Peptides

In vitro pull-down assays with recombinant histones indicated that full-length Pygo proteins (mPygo1, hPygo2, Drosophila was essentially undetectable, like that to other methylated histone tail peptides (H3K9me3 and H4K2Ome3; Figures $3 \mathrm{~A}$ and $3 B)$. The PHD finger from hPygo2 also binds to H3K4me3, as shown by preliminary results with NMR spectroscopy (M.F., J.-C. Yang, and D. Neuhaus, unpublished data). However, we were unable to detect $\mathrm{H} 3 \mathrm{~K} 4 \mathrm{me}$ binding with bacterially expressed dPHD (Figure 3B). Thus, the human, albeit not the Drosophila, Pygo PHD fingers recognize specifically H3K4 in its methylated state.

Given that the predicted $\mathrm{H} 3 \mathrm{~K} 4$ me-binding pocket is opposite the HD1-interacting PHD loop2 surface (Figure 1E), we also tested whether PHD fingers could bind to H3K4me peptides after complex formation with HD1. This is the case, and, interestingly, the affinities of the human PHD-HD1 complex for each methylated H3K4 peptide are $2 x$ to $3 \times$ higher than that of free hPHD: the highest affinity is seen with $\mathrm{H} 3 \mathrm{~K} 4 \mathrm{me} 2\left(\mathrm{~K}_{\mathrm{d}}, 0.9 \mu \mathrm{M}\right)$, but binding to $\mathrm{H} 3 \mathrm{~K} 4 \mathrm{me} 3$ and $\mathrm{H} 3 \mathrm{~K} 4 \mathrm{me} 1$ is also in the low micromolar range $\left(\mathrm{K}_{\mathrm{d}}, 1.2 \mu \mathrm{M}\right.$ and $2.9 \mu \mathrm{M}$, respectively) (Figures $3 \mathrm{~A}$ and $3 \mathrm{~B}$ ).

The effect of PHD-HD1 complex formation on H3K4me binding is even more pronounced in the case of Drosophila: recall that we were unable to detect binding of free $\mathrm{dPHD}$ to H3K4me; however, the dPHD-HD1 complex shows relatively strong affinities for each methylated $\mathrm{H} 3 \mathrm{~K} 4$ peptide and, again, a preference for $\mathrm{H} 3 \mathrm{~K} 4 \mathrm{me} 2\left(\mathrm{~K}_{\mathrm{d}}, 13 \mu \mathrm{M}\right.$ for H3K4me2, $23 \mu \mathrm{M}$ for H3K4me3, and $22 \mu \mathrm{M}$ for H3K4me1). Neither complex showed detectable binding to $\mathrm{H} 3 \mathrm{~K} 4 \mathrm{me}$ (Figures $3 \mathrm{~A}$ and $3 \mathrm{~B}$ ). Therefore, the association of HD1 with human and Drosophila PHD fingers enhances their affinities for methylated H3K4 peptides and reveals their modest preference for $\mathrm{H} 3 \mathrm{~K} 4 \mathrm{me} 2$. 

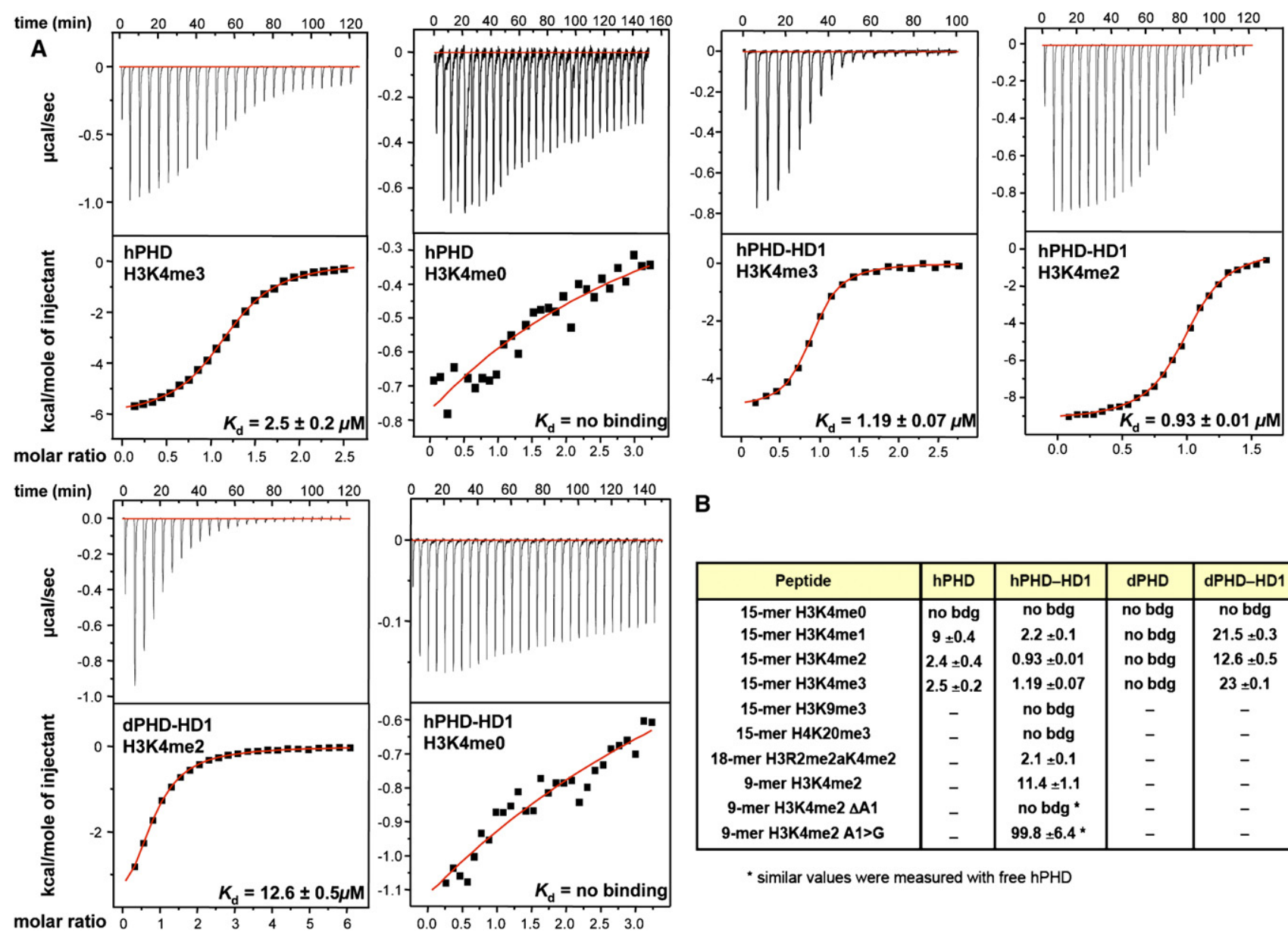

B

\begin{tabular}{|c|c|c|c|c|}
\hline Peptide & hPHD & hPHD-HD1 & dPHD & dPHD-HD1 \\
\hline 15-mer H3K4me0 & no bdg & no bdg & no bdg & no bdg \\
15-mer H3K4me1 & $9 \pm 0.4$ & $2.2 \pm 0.1$ & no bdg & $21.5 \pm 0.3$ \\
15-mer H3K4me2 & $2.4 \pm 0.4$ & $0.93 \pm 0.01$ & no bdg & $12.6 \pm 0.5$ \\
15-mer H3K4me3 & $2.5 \pm 0.2$ & $1.19 \pm 0.07$ & no bdg & $23 \pm 0.1$ \\
15-mer H3K9me3 & - & no bdg & - & - \\
15-mer H4K20me3 & - & no bdg & - & - \\
18-mer H3R2me2aK4me2 & - & $2.1 \pm 0.1$ & - & - \\
9-mer H3K4me2 & - & $11.4 \pm 1.1$ & - & - \\
9-mer H3K4me2 $\Delta$ A1 & - & no bdg * & - & - \\
9-mer H3K4me2 A1>G & - & $99.8 \pm 6.4 *$ & - & - \\
\hline
\end{tabular}

* similar values were measured with free hPHD

Figure 3. Binding Affinities between Histone Peptides and PHD or PHD-HD1

(A) ITC profiles for the binding of methylated and unmodified 15-mer histone H3 tail peptides to free hPHD, hPHD-HD1, or dPHD-HD1 complex, as indicated in the panels; data were fitted to a one-site model. $\mathrm{K}_{\mathrm{d}}$ values are given in the individual panels (with fitting errors indicated; see the Supplemental Experimental Procedures).

(B) Binding constants $\left(\mathrm{K}_{\mathrm{d}}\right.$ values in $\left.\mu \mathrm{M}\right)$ of free human and Drosophila PHD finger versus PHD-HD1 complex for various histone ligands, as indicated.

The Structure of the Ternary Complex

In one of our PHD-HD1 crystals (space group C222, WT2; Table 2), where two binary complexes are seen in the asymmetric unit, one of the N-terminal hPHD ends was inserted into the peptide-binding pocket of the second complex, thus behaving as a pseudoligand (Figure S3). We truncated the $\mathrm{N}$ terminus of $\mathrm{hPHD}$ by seven residues to avoid this autoinhibition artifact, and crystallized this hPHD-HD1 complex with a $5 \times$ molar excess of a 9-mer H3K4me2 peptide ( $K_{d}, 11 \mu \mathrm{M}$; Figure $\left.3 \mathrm{~B}\right)$, which allowed us to solve the structure of this ternary complex (Tern1) at $1.7 \AA$ A resolution (Table 2). We subsequently crystallized the same PHD-HD1 complex with an 18-mer histone H3 tail carrying both $\mathrm{K} 4 \mathrm{me} 2$ and asymmetrically methylated R2 (R2me2a) $\left(\mathrm{K}_{\mathrm{d}}, 2.1 \mu \mathrm{M}\right.$; Figure 3B), and solved the structure of this alternative ternary complex (Tern2) at $1.60 \AA$ resolution. The latter is virtually identical to the structure of Tern1 (with an rmsd for the $\mathrm{C} \alpha$ backbone of $0.16 \AA$; Table 2) described below.

In both maps, we observed additional electron densities in the predicted histone-binding pockets of hPHD, which allowed us to model the first seven amino acids of the histone $\mathrm{H} 3$ tail bound to the hPHD-HD1 complex (Figure 4A; Figure S4). The first five amino acids (A1-Q5) exhibit an extended conformation, with R2-K4me2 forming an antiparallel $\beta$ sheet with PHD B3 (A356-L358) (Figure S5), as observed in all other known PHD-H3K4me structures, while T6 and A7 meander off the PHD surface, looping back on itself, stabilized by an $\mathrm{H}$ bond between T6 and T3 (see also Ramon-Maiques et al., 2007). K4me2 and A1 occupy two adjacent cavities, separated by PHD W366 (Figures 1E and 4A).

The K4me2 cavity consists of a semiaromatic cage with W366, Y341, and D352 forming the walls, and V350 and A356 the base (Figure 4B). The two K4me2 methyl groups make hydrophobic interactions with W366 (in PHD $\beta 4$ ) and Y341, while D352 (in the EVND motif) forms an $\mathrm{H}$ bond with the $\mathrm{N} \zeta$ of the $\mathrm{K} 4 \mathrm{me} 2$ side chain. This $\mathrm{H}$ bond could not be formed with $\mathrm{K} 4 \mathrm{me} 3$ because the extra methyl group would preclude the approach of the D352 carboxyl group. The K4me2 cavity is different from that in BPTF, ING2/Yng1, and RAG2 that prefer K4me3, but 


\begin{tabular}{|c|c|c|c|c|}
\hline Crystal & WT1 Binary & WT2 Binary & Tern1 & Tern2 \\
\hline Beamline & ESRF, ID14-1 & ESRF, ID14-3 & Diamond, 103 & ESRF, ID14-1 \\
\hline Strategy & $225^{\circ}, \Delta \varphi 0.5^{\circ}$ & $180^{\circ}, \Delta \varphi 0.5^{\circ}$ & $135^{\circ}, \Delta \varphi 0.5^{\circ}$ & $90^{\circ}, \Delta \varphi 0.5^{\circ}$ \\
\hline Wavelength $(\AA)$ & 0.934 & 0.931 & 1.06 & 0.934 \\
\hline Space Group & 1222 & $\mathrm{C} 222_{1}$ & $\mathrm{P} 42_{1} 2$ & $\mathrm{P} 42_{1} 2$ \\
\hline a, b, c (Å) & $32.10,79.47,91.83$ & $88.72,100.89,64.24$ & $105.72,105.72,51.43$ & $105.75,105.75,51.42$ \\
\hline$\alpha, \beta, \gamma\left({ }^{\circ}\right)$ & $90,90,90$ & $90,90,90$ & $90,90,90$ & $90,90,90$ \\
\hline Resolution $(\AA ̊)$ & $28.56-1.59(1.68-1.59)^{a}$ & $31.450-2.77(2.92-2.77)$ & $42.37-1.70(1.79-1.70)$ & $26.44-1.6(1.69-1.60)$ \\
\hline$R_{\text {merge }}(\%)^{b}$ & $5.6(37.4)$ & $6.3(37.7)$ & $11.8(121.6)$ & $11.7(60.1)$ \\
\hline $1 / \sigma(I)$ & $26.6(11.4)$ & $22.5(4.2)$ & $12.4(2.2)$ & $11.4(3.1)$ \\
\hline Completeness (\%) & 99.9 (99.9) & 99.7 (99.7) & $100(100)$ & $100(100)$ \\
\hline Multiplicity & $10.5(10.3)$ & $7.0(6.9)$ & $10.3(10.2)$ & $7.1(7.0)$ \\
\hline Complexes in A.U. & 1 & 2 & 2 & 2 \\
\hline \multicolumn{5}{|l|}{ Refinement } \\
\hline Resolution $(\AA)$ & $28.56-1.59$ (1.63-1.59) & $31.450-2.77(2.84-2.77)$ & $42.37-1.70(1.79-1.70)$ & $26.44-1.60(1.64-1.60)$ \\
\hline Number of reflections & 15,426 & 7263 & 30,994 & 37,079 \\
\hline Test set size (\%) & 5.0 & 4.6 & 5.1 & 5.0 \\
\hline $\mathrm{R}_{\text {work }}(\%)$ & $19.2(18.6)$ & $23.3(29.5)$ & $19.3(29.5)$ & $20.1(27.2)$ \\
\hline $\mathrm{R}_{\text {free }}(\%)$ & $22.5(19.6)$ & $26.7(42.4)$ & $23.0(38.1)$ & $21.8(32.1)$ \\
\hline Number of atoms (non-H) & 812 & 1473 & 1688 & 1759 \\
\hline Residues (PHD/HD1) & 341-397/173-205 & 333-397/174-205 & 339-398/174-203 & 339-398/174-203 \\
\hline$<B>\left(\AA^{2}\right)$ & 22.9 & 64.8 & 28.8 & 22.0 \\
\hline \multicolumn{5}{|l|}{ Rmsd } \\
\hline Bond length $(\AA)$ & 0.010 & 0.008 & 0.011 & 0.010 \\
\hline Bond angle $\left({ }^{\circ}\right)$ & 1.147 & 0.986 & 1.197 & 1.210 \\
\hline \multicolumn{5}{|l|}{ Ramachandran plot } \\
\hline In favored regions (\%) & 97.8 & 94.1 & 97.8 & 97.2 \\
\hline In allowed regions (\%) & 1.1 & 4.8 & 2.2 & 2.8 \\
\hline Outliers (\%) & $1.1\left(\mathrm{~S} 362^{\mathrm{C}}\right)$ & $1.1\left(\mathrm{~S} 362^{\mathrm{C}}\right)$ & 0.0 & 0.0 \\
\hline
\end{tabular}

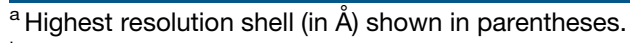

${ }^{b} R_{\text {merge }}=\Sigma_{\text {hkl }}\left|l_{\text {hkl }}-<l_{\text {hkl }}\right| / \Sigma_{\text {hkl }} I_{\text {hkl }}$.

${ }^{\mathrm{C}} \mathrm{S} 362$ presents an unusual conformation (the electron density map is clear in this area) because it is next to the C363, which is part of the second $\mathrm{Zn}^{2+}$-binding site (Zn2).

reminiscent of that in the $\mathrm{H} 3 \mathrm{~K} 4 \mathrm{meO}$-binding $\mathrm{PHD}$ finger of $\mathrm{BHC} 80$ (see the Discussion).

The adjacent cavity is deep and accommodates A1 (instead of R2; see below). Specifically, the N-terminal amino group of $A 1$ is tightly bound to backbone carbonyl groups of L382, E385, and A388 (Figure 4C), as in all other PHD fingers. A1 is further stabilized through hydrophobic interactions with L382 and V389 mediated by its $C \beta$ side chain. Notably, all these PHD residues (in $\alpha 1$ or $\beta 5$ ) interact simultaneously with HD1 (Figure 2; see also below).

R2 and T3 are located between these two cavities, with the methyl group of T3 buried in a pocket formed by A356, I357, W366, and $\mathrm{Y} 379$, and its $\mathrm{OH}$ forming an $\mathrm{H}$ bond to T383 (Figure S5). Interestingly, although $\mathrm{R} 2$ forms two $\mathrm{H}$ bonds through its backbone with L358 and with the carboxyl group of the invariant E360, as well as hydrophobic side-chain interactions with L358 and W366 (Figure S5), its charged guanidinium group protrudes from the PHD surface into the solvent (Figure 4D), and adopts multiple conformations in one of the complexes of the asymmetric unit of Tern1. The same is observed in Tern2 (data not shown) in which the methylated guanidinium group of R2 is not able to assume a stable conformation, due to lack of molecular contacts with the PHD finger. This is different from the PHD fingers of BPTF and ING2/Yng1 where R2 is entirely buried in a pronounced pocket adjacent to the K4me cavity. In fact, there is no discernible R2 pocket in the Pygo PHD finger; instead, the long hydrophobic side chain of L358 protrudes into the groove corresponding to the R2 pocket in other PHD fingers, blocking access of R2's side chain and redirecting it into the solvent (Figure 4D). This is somewhat reminiscent of the RAG2 PHD finger, where a tyrosine protrudes into the R2 pocket and interacts with the methyl groups of symmetrically methylated R2 (R2me2s)though not with $\mathrm{R} 2 \mathrm{me} 2 \mathrm{a}$-thereby stabilizing the conformation of R2 (Ramon-Maiques et al., 2007).

\section{HD1 Buttresses the Bottom of the PHD A1 Cavity from the Back}

Superimposition of our structures of the PHD-HD1 complex on that of the free hPHD finger (Nakamura et al., 2007) shows that 

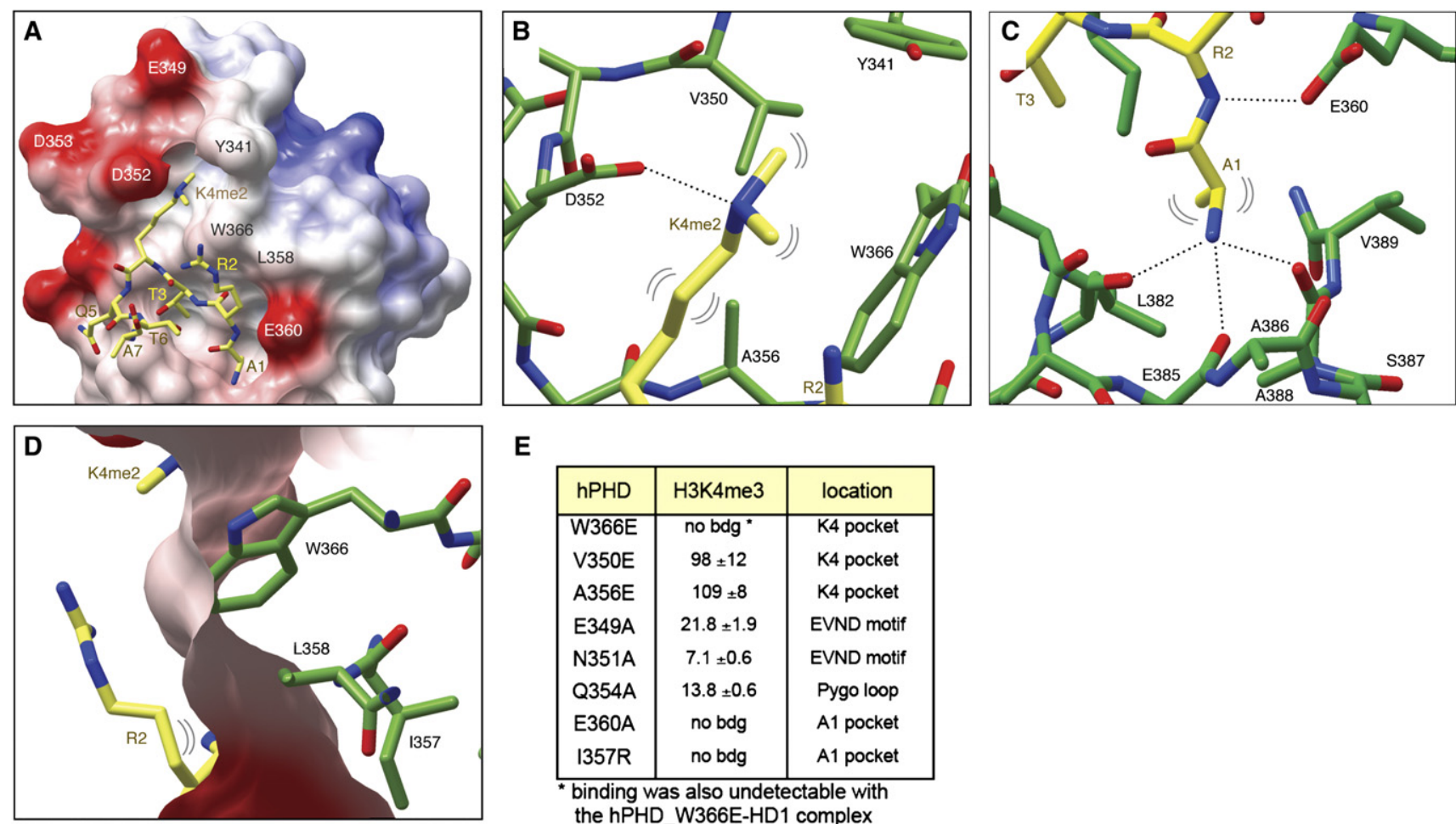

E

\begin{tabular}{|c|c|c|}
\hline hPHD & H3K4me3 & location \\
\hline W366E & no bdg * & K4 pocket \\
\hline V350E & $98 \pm 12$ & K4 pocket \\
\hline A356E & $109 \pm 8$ & K4 pocket \\
\hline E349A & $21.8 \pm 1.9$ & EVND motif \\
\hline N351A & $7.1 \pm 0.6$ & EVND motif \\
\hline Q354A & $13.8 \pm 0.6$ & Pygo loop \\
\hline $\mathrm{E} 360 \mathrm{~A}$ & no bdg & A1 pocket \\
\hline I357R & no bdg & A1 pocket \\
\hline
\end{tabular}

Figure 4. Structures of the Ternary Complex, and H3K4me-Binding Cavities

(A) Molecular surface representation of hPHD-HD1 binding to H3K4me2 (in yellow cylinder style), with W366 and other critical residues labeled. ( $B$ and $C$ ) Cylinder representations of (B) semiaromatic K4me2 cavity and (C) A1 cavity, with critical $\mathrm{H}$ bonds indicated as dotted lines and hydrophobic contacts as double brackets.

(D) Molecular surface representation of PHD, revealing solvent exposure of R2 (regardless of its methylation status). H3K4me, yellow; PHD cavity residues, green. Note that Tern2 has essentially the same structure as Tern1 (shown here; see text).

(E) Binding constants of various hPHD point mutants for H3K4me3 15-mer ( $\mathrm{K}_{d}$ values in $\mu \mathrm{M}$; see also Figure 3).

the structures are very similar ( $r m s d$ for the $\mathrm{C} \alpha$ backbone, $0.6 \AA$ ), except for small local conformational changes of two short loop segments.

One of these is the Pygo loop centered on D352, which constitutes one of the walls of the K4me2 pocket (Figure S6A, arrow 1). This loop is flexible, and although its backbone is clearly defined, the electron density for the side chains in some cases is poor. Its conformation varies between the various structures and indeed is different in the two independent molecules of the free PHD ( $\mathrm{Na}$ kamura et al., 2007). In the ternary complexes, the PHD groups around the $\mathrm{K} 4 \mathrm{me} 2$ pocket close up slightly around the peptide (Figure S6B, arrow 1) and the Pygo loop becomes more ordered.

The second difference is in the PHD-HD1 interface, in a loop segment between $\alpha 1$ and $\beta 5$ (L382-S387). This loop is pushed out in the binary complex compared to the free PHD finger (Figure S6A, arrow 2), as a direct consequence of the PHDHD1 interactions (Figure 2), opening up the A1 cavity in the complex. On binding the peptide, there are only small shifts in the residues lining the $A 1$ pocket. Interestingly, these residues that contact $A 1$ are also engaged in contacting HD1, contributing to the PHD-HD1 interface (Figures 2, 4C, and 4D). The base of the $A 1$ cavity is very thin (Figure $5 \mathrm{~A}$ ) and is buttressed from behind by HD1 (Figure 5B). Thus, the PHD-HD1 interactions are likely to shape and stabilize the A1 cavity (see the Discussion).
There is also an apparent conformational change of the HD1 C-terminal tail in the binary versus ternary complex (Figure S6B). However, we believe that the conformation seen in the ternary complex is a crystal artifact, given that there are multiple direct contacts between PHD $\alpha 1$ and $\beta 5$ and the invariant I200 and semiconserved V201, both included in the minimal PHD-binding fragment of HD1 (Figure S2).

\section{Function of the H3K4me Pockets in Binding Histone Н3 Tail}

We conducted ITC binding studies of hPHD with point mutations designed to alter the two cavities, to gain experimental support for their function in $\mathrm{H} 3 \mathrm{~K} 4 \mathrm{me}$ binding. To test the K4me cavity, we mutated W366 (which forms its proximal wall), and V350 and $\mathrm{A} 356$ (which undergo side-chain interactions with $\mathrm{K} 4 \mathrm{me} 2$; Figure 4B). No binding whatsoever was detectable with W366E, and V350E and A356E strongly diminished the affinity to $\mathrm{H} 3 \mathrm{~K} 4 \mathrm{me}$, to below $3 \%$ of the wild-type (WT) hPHD (Figure 4E). These three residues are therefore critical for ligand recognition. Notably, complex formation of these PHD mutants with HD1 is normal (Figure S1D; data not shown), consistent with our structural data that distinct portions of the PHD-HD1 complex are devoted to the K4 pocket and the HD1-interacting surface, respectively. 
A

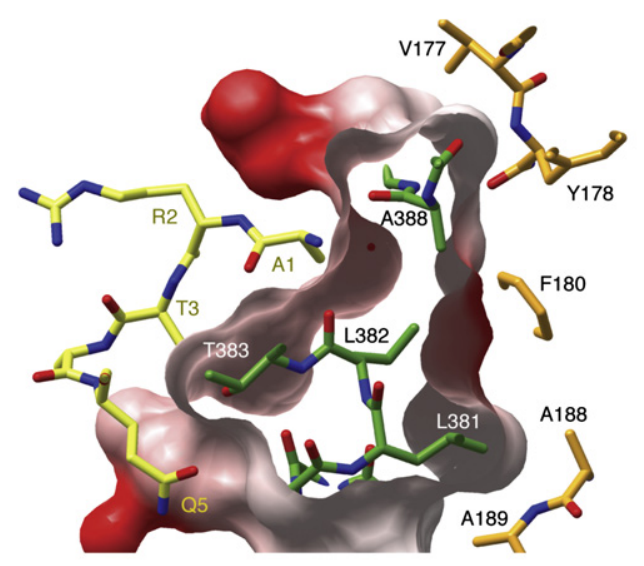

B

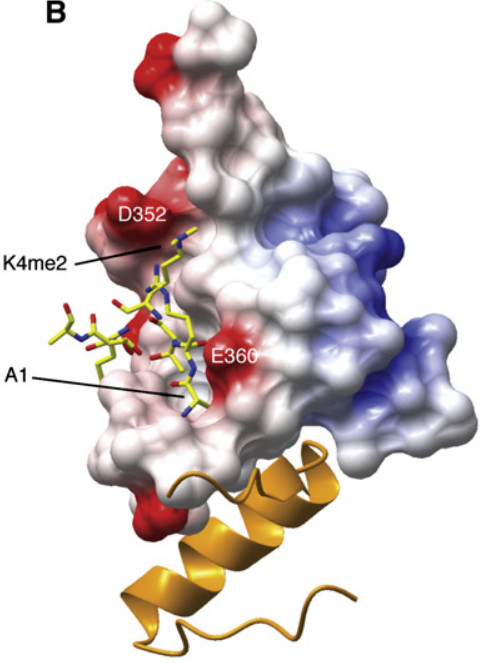

Figure 5. Buttressing of the PHD A1 Cavity by HD1

(A) Molecular surface representation of PHD (green) with electrostatic potential, facing A1 cavity (left, yellow) and HD1 (right, orange).

(B) Buttressing of A1 cavity of PHD (molecular surface representation with electrostatic potential) by HD1 (ribbon representation). D352 lip residue of the K4me2 cavity and $\mathrm{E} 360$ residue critical for $\mathrm{A} 1$ anchoring are indicated.
Testing the function of the A1 cavity by mutational analysis appeared more challenging because its key residues mostly undergo backbone interactions with the ligand. Nevertheless, we decided to mutate the invariant E360 and 1357 that are engaged in direct contacts with A1 and T3, respectively (Figure 4C, Figure S5). Each mutant showed only background levels of $\mathrm{H} 3 \mathrm{~K} 4 \mathrm{me} 3$ binding (Figure 4E), showing that the structural integrity of the $A 1$ pocket is essential for the binding to the methylated histone $\mathrm{H} 3$ tail.

To further confirm the importance of the A1 pocket, we measured the affinities of PHD-HD1 to a truncated H3K4me2 peptide spanning R2-K9 (without $A 1, \Delta A 1$ ), and of a more subtly mutated 9-mer whose $A 1$ was substituted with a glycine $(A 1>G)$. No binding was detectable with $\Delta \mathrm{A} 1$, and the affinity of $\mathrm{A} 1>\mathrm{G}$ to PHD-HD1 was also reduced to almost background levels (Figure 3B). Evidently, the $\mathrm{N}$-terminal $\mathrm{A} 1$ residue of histone $\mathrm{H} 3$ is critical for anchoring of its tail in the PHD finger.

Finally, we tested the functional contribution of the Pygo loop to histone binding, by mutating the EVND residues E349 and N351 and the semiconserved Q354 (Figure 4A). The latter is of particular interest because the corresponding residue in Drosophila Pygo (D761) proved to be the most critical of a series of individually mutated residues in transient transcription assays in mammalian cells (Townsley et al., 2004b). Indeed, each individual point mutation reduced ligand binding, with the worst affected mutant (E349A) retaining only $\sim 11 \%$ of the WT affinity $\left(K_{d}, 22\right.$ $\mu \mathrm{M}$; Figure 4E). These Pygo loop residues thus seem to contribute to ligand binding despite their lack of direct contact with H3K4me2 (Figure 4A).

\section{An Intact H3K4me-Binding Pocket Is Critical} for Pygo Function during Drosophila Development

To test whether the binding of Pygo to H3K4me is important for its function in vivo, we conducted rescue assays in Drosophila (de la Roche and Bienz, 2007). We introduced a point mutation into V757 (in EVND) of Drosophila Pygo, which corresponds to V350 of hPHD that lines its K4me pocket (Figure 4B) and whose mutation drastically reduces its binding to $\mathrm{H} 3 \mathrm{~K} 4 \mathrm{me} 3$ (Figure 4E). We then generated pygo null mutant clones in wing discs, one of the best-characterized Wingless-responsive tissues whose
Overexpression of WT Pygo restored expression of senseless, a well-established Wingless target gene in the developing wing discs (de la Roche and Bienz, 2007; Parker et al., 2002), in pygo null mutant wing disc clones (Figures $6 \mathrm{~A}$ and $6 \mathrm{~B}$ ). However, no rescue whatsoever was observed after overexpression of Pygo-V757E in these clones (Figure 6C), although this mutant was expressed at levels comparable to WT Pygo (Figure 6B). Likewise, WT Pygo but not Pygo-V757E rescued all pygo mutant phenotypes (i.e., notches and bristle defects) in the anterior wing margin of flies (Figures 6D-6F). We conclude that an intact K4me2-binding pocket is critical for Pygo function during Drosophila development.

\section{DISCUSSION}

Our work indicates a physiological role of Pygo proteins in decoding the methylation status of the histone $\mathrm{H} 3$ tail, which is critical for the regulation of Wnt-induced transcription. Based on our biochemical and structural evidence, we discuss how Pygo, assisted by BCL9/Lgs as its cofactor, achieves a unique specificity in decoding the combined methylation status at R2 and $\mathrm{K} 4$ on the histone $\mathrm{H} 3$ tail.

\section{The Interaction between Pygo and BCL9}

Our structures of the PHD-HD1 complex identify the PHD loop2 segment as the BCL9-interacting surface, with a relatively flat and hydrophobic nature. The interaction has two parts, a parallel $\beta$ sheet between strands PHD $\beta 5$ and HD1 $\beta 1$, and a hydrophobic core between the two helices. The buried surface area is relatively small ( $\sim 1240 \AA^{2}, 15 \%$ and $18 \%$ of the total solvent-accessible surface areas of PHD and HD1, respectively), which is consistent with a relatively transient interaction, as might be expected for a signaling molecule. These structural data are fully consistent with our previous functional analysis during Drosophila development (see above; Townsley et al., 2004b).

The structure of the free hPHD finger (Nakamura et al., 2007) shows a dimer in the crystal, in which the PHD-PHD interaction mimics the PHD-HD1 interaction that we see. This PHD-PHD interaction may thus merely reflect a propensity of the 


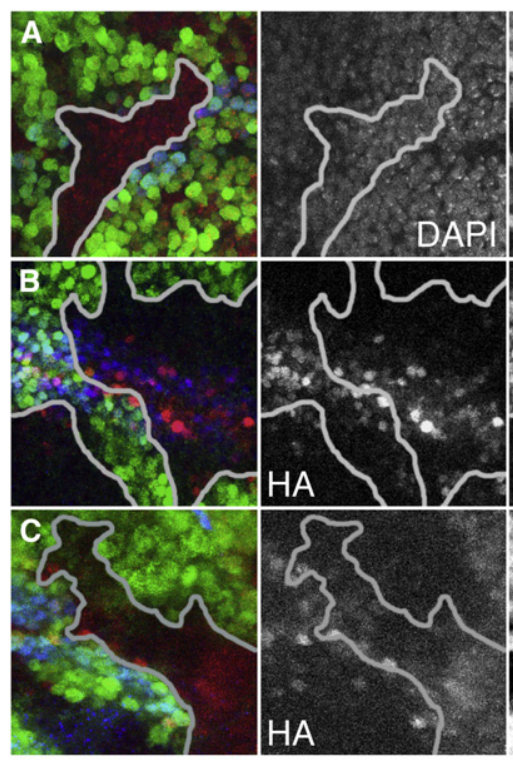

merge
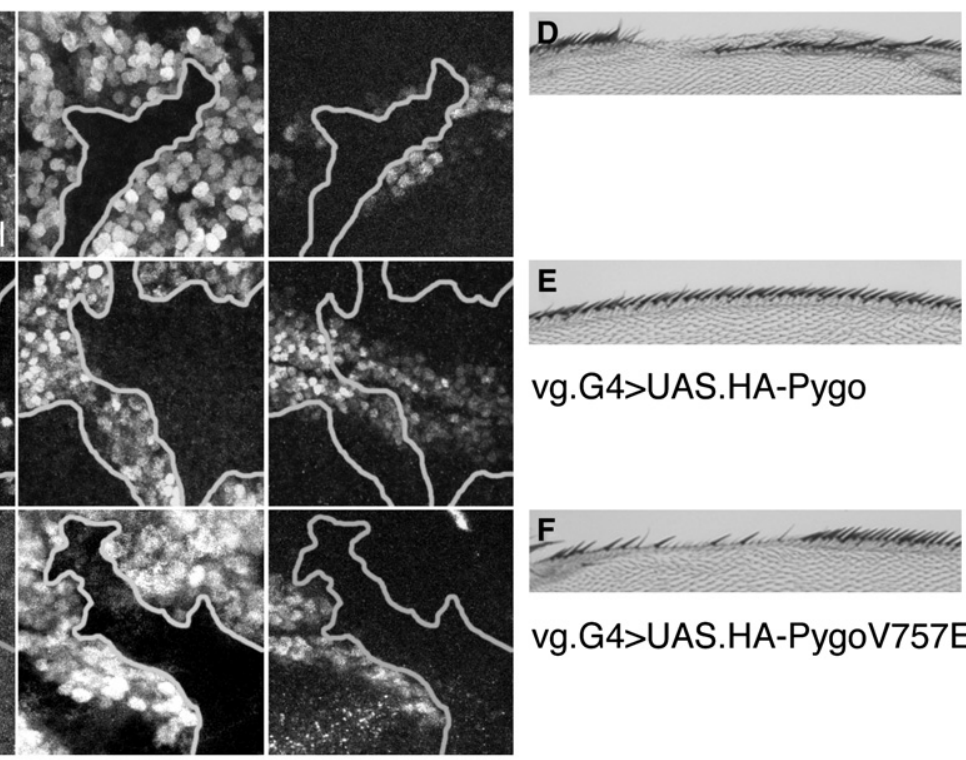

Senseless

Figure 6. Rescue Assays in Drosophila, Revealing Functional Relevance of K4me Cavity

(A-C) Wing discs of third instar larvae bearing pygo ${ }^{S 28}$ mutant clones (marked by absence of GFP), with or without overexpressed HA-Pygo, double stained with (A) DAPI (to reveal the nuclei) or (B and C) anti-HA antibody, and (A-C) anti-Senseless antibodies.

(D-F) Anterior wing margin of adult flies bearing pygo ${ }^{S 28}$ mutant clones, with or without overexpressed HA-Pygo. (A and D) controls, (B and E) WT HA-Pygo, (C and F) HA-Pygo-V757E.

hydrophobic loop2 surface to undergo a pseudoligand interaction in the absence of bona fide ligand, similar to the pseudoligand interaction we observed between the hPHD N terminus and the H3K4me-binding pocket (Figure S3). We note that Pygo dimerization is undetectable in vivo (M.F., unpublished data) and so may not be physiologically relevant.

\section{BCL9 Binding to Pygo Boosts Its Recognition of Methylated Histone H3 Tails}

Perhaps our most interesting finding was that the binding of BCL9 HD1 to Pygo PHD increases its affinity to H3K4me. This is particularly striking in Drosophila where the H3K4me binding of the Pygo PHD finger depends entirely on its interaction with Lgs HD1 (Figure 3). Given that the backbone structures of human and Drosophila PHD-HD1 complexes are very similar (M.F., M.J.S.-B., and M.B., unpublished data), it seems likely that the underlying principle for this boosting effect of HD1 on the H3K4me recognition by $\mathrm{PHD}$ is essentially the same in both species.

How does HD1 enhance the H3K4me binding of PHD? The answer is found in the structure of the A1 cavity whose floor is very thin and formed by PHD loop2 residues that are simultaneously engaged in direct interactions with HD1. Indeed, this floor is formed largely by the C-terminal $\beta$ strand of the PHD finger (Figures 2, 4, and 5A), and although this PHD segment appears to be well structured in the absence of HD1 (Nakamura et al., 2007), this may be partly because the observed PHD-PHD interactions in the crystal mimic the PHD-HD1 contacts. It is therefore possible that the PHD C terminus is somewhat floppy in the absence of HD1, so that its buttressing by HD1 may contribute to the shape and/or stability of the cavity that is required for firm an- choring of the $\mathrm{N}$-terminal alanine residue of the histone $\mathrm{H} 3$ tail. Recall that this residue, and the structural integrity of the A1 cavity, are essential for the binding of $\mathrm{H} 3 \mathrm{~K} 4 \mathrm{me}$ (Figures 3B and 4E).

\section{Histone H3 Tail Recognition}

Recent work indicates that the transition from transcriptional silence to activation of a gene involves a change in the methylation state of (at least) two side chains on the histone H3 tail-R2 and K4 (Guccione et al., 2007; Kirmizis et al., 2007). This is monitored by PHD proteins that bind to this tail, and specific examples that decode the methylation status of $\mathrm{K} 4$ have been discovered. Comparison of the structures now available (Lan et al., 2007; Li et al., 2006; Pena et al., 2006; Taverna et al., 2006) suggests how this is done. Namely, the recognition of the H3 tail by PHD fingers depends on two sets of interactions: those that involve a constant $\mathrm{H} 3$ segment and provide much of the binding affinity, and those that are sensitive to the methylation status of $R 2$ and $\mathrm{K} 4$ and thus provide specificity.

The former set of interactions is similar in all known PHD fingers and comprises (1) the N-terminal alanine of the $\mathrm{H} 3$ tail in its deep PHD pocket, which accommodates both the alanine side chain and the terminal $\mathrm{NH}_{3}{ }^{+}$group; (2) the T3 side chain, with a hydrophobic pocket and an $\mathrm{H}$ bond to recognize the side chain; and (3) the main $\mathrm{H} 3$ peptide chain, which forms a short $\beta$ sheet with the PHD. Recall that the binding of HD1 to Pygo PHD fingers affects primarily this set of interactions, thus boosting the affinity between $\mathrm{PHD}$ and histone $\mathrm{H} 3$ tail. Other PHD proteins such as BPTF, ING2/Yng1, and BHC80 are subunits of transcription complexes, but it is not known whether similar partner interactions control their binding to modified histone ligands. We 
note, though, that there are precedents, largely in the context of DNA repair, of different protein modules (e.g., Royal superfamily domains) that cooperate in the binding to modified histone tails (Corsini and Sattler, 2007; Taverna et al., 2007).

Pygo PHD fingers are distinguished from other PHD fingers with regard to the second set of interactions that serve to decode the methylation status of the histone $\mathrm{H} 3$ tail. First, they show a modest preference for $\mathrm{H} 3 \mathrm{~K} 4 \mathrm{me} 2$, which can be explained by the structure of the K4me2 cavity of the PHD-HD1 complex, in particular by the $\mathrm{H}$ bond between the pocket lip residue D352 and the side-chain amino group of K4me2 that would not be able to form with K4me3 (Figure 4B). A similar salt bridge with an aspartate residue is observed in the $\mathrm{K} 4 \mathrm{meO}$-binding pocket of BHC80 (Lan et al., 2007), and also in structurally unrelated histone-binding domains with a preference for hypomethylated lysines in various histone tail contexts, such as MBT domains (Grimm et al., 2007; Li et al., 2007b) and the tandem-Tudor domain (Botuyan et al., 2006). By contrast, the PHD fingers with preference for K4me3 contain either a full aromatic cage (BTPF) or semiaromatic, semihydrophobic cages (ING2/Yng1) that provide optimal accommodation of $\mathrm{K} 4 \mathrm{me} 3$ through multiple hydrophobic side-chain interactions. Modeling of the K4me3 ligand in the Pygo K4me cavity suggests that this ligand would slightly displace the D352 lip residue in the Pygo loop; however, the high flexibility of this loop would accommodate this. Evidently, such a displacement can be tolerated without much loss of binding affinity (Figure 3), no doubt due to the numerous additional interactions between PHD and histone $\mathrm{H} 3$ tail peptide. Especially critical may be the hydrophobic side-chain interaction between one of the K4-methyl groups and the aromatic cavity wall residue W366 (Figure 4B), which is essential for H3K4me binding (Figure 4E), and its interaction with the K4-methyl group(s) may thus be pivotal in discriminating methylated from unmodified $\mathrm{H} 3 \mathrm{~K} 4$ tails.

The second distinct feature relates to H3R2: in the BPTF and ING2/Yng1 PHD fingers, R2 is completely anchored by its guanidinium group within a groove adjacent to the K4me-binding pocket (Li et al., 2006; Pena et al., 2006; Taverna et al., 2006), which would be incompatible with methylation. Indeed, the H3K4me binding of the SET1 PHD finger is blocked by R2me2a (Kirmizis et al., 2007). However, in both our ternary complexes, only the first two carbons of the R2 side chain are fixed (Figure 4C), while the guanidinium group at the end of this side chain is pushed out into the solvent by the side chain of L358 (Figure 4D), regardless of the methylation status of R2. Taken together with our binding data that revealed insensitivity to R2 methylation, this implies that Pygo PHD fingers recognize equally well histone $\mathrm{H} 3$ tails that are methylated or unmodified at R2. Interestingly, R2me2a appears to be linked to transcriptional silence, and is mutually exclusive with $\mathrm{H} 3 \mathrm{~K} 4 \mathrm{me} 3$ in chromatin (Guccione et al., 2007; Kirmizis et al., 2007), which itself is a histone mark for gene activity (Kouzarides, 2007).

These two unique structural features of Pygo's H3K4mebinding pocket enable it to recognize histone $\mathrm{H} 3$ tails that bear both K4me2 and R2me2a marks. The combined occurrence of these two chromatin marks is indicative of an early step in the transition from gene silence to gene activation: evidence suggests that, for a gene to become fully active, H3R2me2a needs to be demethylated before H3K4 can be trimethylated by SET1 methyltransferases (Guccione et al., 2007; Kirmizis et al., 2007).

Based on our structural analysis, we propose that Pygo plays a role early during the transition from gene silence to Wnt-induced gene activation (e.g., in step 1 of the proposed sequence of events; Kirmizis et al., 2007). Indeed, the Pygo-BCL9 complex may function to expose H3R2me2a for demethylation (by an as-yet-unknown enzyme), prior to recruiting the SET1 methyltransferase complex (Sierra et al., 2006) through capturing $\beta$-catenin at TCF target genes during incipient Wnt signaling (de la Roche and Bienz, 2007; Townsley et al., 2004a). SET1 recruitment would then result in the conversion of $\mathrm{H} 3 \mathrm{~K} 4 \mathrm{me} 2$ to H3K4me3, which in turn would allow full transcriptional activity of TCF target genes during sustained Wnt signaling.

\section{The Potential of the Pygo H3K4me-Binding Pockets as Targets for Small-Molecule Inhibitors}

Despite the importance of the Wnt pathway in cancer, there are no well-established small-molecule inhibitors of this pathway to date, probably because the oncogenic mutations that cause pathway hyperactivation typically occur at the level of, or immediately above, $\beta$-catenin (Bienz and Clevers, 2000; Polakis, 2000). However, there are no enzymes to inhibit below activated $\beta$-catenin, and the protein interaction surface between $\beta$-catenin and TCF is highly unsuitable as a drug target due to its extensive nature and multiple overlapping interactions with negative regulators of the Wnt pathway (Daniels et al., 2001).

The function of the Pygo-BCL9 complex in Wnt signaling depends on at least three physiologically relevant protein-protein interactions: those between PHD and its two ligands (HD1 and histone $\mathrm{H} 3$ tail) described here, and that between BCL9 HD2 and $\beta$-catenin (Sampietro et al., 2006). Each of these is known at the structural level and is sensitive to point mutations of single residues (Sampietro et al., 2006; Townsley et al., 2004b; this work), suggesting that they may have potential as targets for disruption by small compounds.

\section{EXPERIMENTAL PROCEDURES}

Expression and Purification of Proteins, and ITC Measurements hPygo1 PHD (amino acids 333-402) or Drosophila Pygo PHD (amino acids 747-808) fused to GST, and hBCL9 HD1 (amino acids 177-205) or Lgs HD1 (amino acids 316-350) fused to MBP, were coexpressed in a bicistronic expression vector (including N-terminal TEV protease sites for removal of tags) in E. coli BL21-CodonPlus(DE3)-RIL cells (Stratagene), and PHD-HD1 complexes were purified as described in the Supplemental Experimental Procedures (see also Figure S1). Free PHD fingers were purified similarly to the PHD-HD1 complexes, and purity was assessed by SDS-PAGE prior to use for ITC. ITC was performed after dialysis of protein into $100 \mathrm{mM} \mathrm{NaCl}$, $25 \mathrm{mM}$ Tris- $\mathrm{HCl}$ ( $\mathrm{pH}$ 8.0), $10 \mu \mathrm{M} \mathrm{ZnCl}_{2}$, and $2 \mathrm{mM} \beta$-mercaptoethanol, essentially as described (Grimm et al., 2007) (see also the Supplemental Experimental Procedures).

\section{Crystallization}

Concentrated protein was centrifuged at $100,000 \times \mathrm{g}$ for $15 \mathrm{~min}$ and used for crystallization (initial screen of 1500 different crystallization conditions in a 96-well sitting drop format using $100 \mathrm{nl}$ drops; Stock et al., 2005). Crystals grown at $19^{\circ} \mathrm{C}$ by the vapor diffusion method emerged after $\sim 24 \mathrm{hr}$ under multiple conditions. The following conditions were used: $1.7 \mathrm{M}\left(\mathrm{NH}_{4}\right)_{2} \mathrm{SO}_{4}, 100 \mathrm{mM}$ Tris (pH 7.5), and $200 \mathrm{mM} \mathrm{NaCl}$ (for hPHD_W366F-HD1); $1 \mathrm{M} \mathrm{Na-citrate,}$ $100 \mathrm{mM}$ Tris $\left(\mathrm{pH} \mathrm{7}\right.$ ), and $200 \mathrm{mM} \mathrm{NaCl}$ (for WT1); and $1.3 \mathrm{M}\left(\mathrm{NH}_{4}\right)_{2} \mathrm{SO}_{4}$, 
$100 \mathrm{mM}$ Tris ( $\mathrm{pH} 6.5$ ), and $200 \mathrm{mM} \mathrm{NaCl}$ (for WT2, space group C222 1 ). For the ternary complexes, $10 \mathrm{mg} / \mathrm{ml}$ of hPHD-HD1 was mixed with $5 \times$ molar excess of H3K4me2 9-mer (Figure 4) or H3R2me2aK4me2 18-mer peptide (1 hr incubation on ice followed 15 min centrifugation at $100,000 \times$ g) prior to crystallization with 26.4\% PEG-3350, $200 \mathrm{mM} \mathrm{LiSO}_{4}$, and $100 \mathrm{mM}$ Tris $(\mathrm{pH} \mathrm{7)}$ ), or $20 \%$ PEG-4000, 20\% isopropanol, and $0.1 \mathrm{M} \mathrm{Na}$ citrate, respectively; for both complexes, amino acids 340-402 of hPHD were used, to avoid blocking of the H3K4me2 pocket (Figure S3). Each crystal was soaked for < 1 min in $25 \%$ glycerol as cryoprotectant before flash-cooling in liquid nitrogen.

\section{Diffraction Data Collection and Structure Solution}

Different diffraction data sets were collected at ESRF and Diamond synchrotrons (Tables 1 and 2). The initial hPHD-HD1 structure was phased using the anomalous signal from the $\mathrm{Zn}^{2+}$ ions binding to PHD. A fluorescence scan was performed to select the wavelengths and collect a MAD data set from a crystal of hPHD_W366F-HD1 (Table 1); the peak was collected first, followed by the inflexion point and the remote. Crystallographic data were processed, and the structure was phased, as described in the supplement. The initial structure of hPHD_W366F-HD1 was used to solve four other crystal forms by molecular replacement with Phaser (Table 2) (McCoy et al., 2005): WT1 (isomorphous with hPHD_W366F-HD1) at higher resolution; WT2, a second crystal form (space group C222 1 ) exhibiting pseudoligand binding (Figure S3); and the two ternary complexes.

\section{Rescue Assays in Drosophila}

HA-tagged Pygo-V757E was constructed in pUAST, and multiple independent transformant lines were isolated, as described previously for WT HA-Pygo (Townsley et al., 2004b). Two different lines were each tested for rescue activity of pygo null mutant clones in the wing disc, as described (de la Roche and Bienz, 2007).

\section{ACCESSION NUMBERS}

Coordinates and structure factors have been deposited with the Protein Data Bank under accession codes 2vp7 (hPHD_W366F-HD1), 2vpb (WT1 Binary), 2vpd (WT2 Binary), 2vpe (Tern1), and 2vpg (Tern2).

\section{SUPPLEMENTAL DATA}

Supplemental Data include Supplemental Experimental Procedures, Supplemental References, and six figures and can be found with this article online at http://www.molecule.org/cgi/content/full/30/4/507/DC1/.

\section{ACKNOWLEDGMENTS}

We thank Olga Perisic and Helen Kent for advice with protein expression, and we thank ESRF (beamlines ID23-1, ID14-1, and ID14-3) and Diamond (beamline I03) synchrotrons for the access to the facilities and help in data collection. M.F. was funded by an Erwin-Schrödinger postdoctoral fellowship, M.J.S.-B. by an FEBS postdoctoral fellowship, and M.N. by an E-STAR Marie Curie fellowship (FP6 number MEST-CT-2004-504640). This work was funded by the Medical Research Council and by Cancer Research UK (grant number C7379/A8709 to M.B.).

Received: November 14, 2007

Revised: January 29, 2008

Accepted: March 11, 2008

Published: May 22, 2008

\section{REFERENCES}

Adachi, S., Jigami, T., Yasui, T., Nakano, T., Ohwada, S., Omori, Y., Sugano, S., Ohkawara, B., Shibuya, H., Nakamura, T., and Akiyama, T. (2004). Role of a BCL9-related $\beta$-catenin-binding protein, B9L, in tumorigenesis induced by aberrant activation of Wnt signaling. Cancer Res. 64, 8496-8501.
Arce, L., Yokoyama, N.N., and Waterman, M.L. (2006). Diversity of LEF/TCF action in development and disease. Oncogene 25, 7492-7504.

Belenkaya, T.Y., Han, C., Standley, H.J., Lin, X., Houston, D.W., Heasman, J., and Lin, X. (2002). pygopus encodes a nuclear protein essential for wingless/ Wnt signaling. Development 129, 4089-4101.

Bienz, M. (2006). The PHD finger, a nuclear protein-interaction domain. Trends Biochem. Sci. 31, 35-40.

Bienz, M., and Clevers, H. (2000). Linking colorectal cancer to Wnt signaling. Cell 103, 311-320.

Botuyan, M.V., Lee, J., Ward, I.M., Kim, J.E., Thompson, J.R., Chen, J., and Mer, G. (2006). Structural basis for the methylation state-specific recognition of histone H4-K20 by 53BP1 and Crb2 in DNA repair. Cell 127, 1361-1373.

Brembeck, F.H., Schwarz-Romond, T., Bakkers, J., Wilhelm, S., Hammerschmidt, M., and Birchmeier, W. (2004). Essential role of BCL9-2 in the switch between $\beta$-catenin's adhesive and transcriptional functions. Genes Dev. 18, 2225-2230.

Clevers, H. (2006). Wnt/ $\beta$-catenin signaling in development and disease. Cell 127, 469-480.

Corsini, L., and Sattler, M. (2007). Tudor hooks up with DNA repair. Nat. Struct. Mol. Biol. 14, 98-99.

Daniels, D.L., Eklof Spink, K., and Weis, W.I. (2001). $\beta$-catenin: molecular plasticity and drug design. Trends Biochem. Sci. 26, 672-678.

de la Roche, M., and Bienz, M. (2007). Wingless-independent association of Pygopus with dTCF target genes. Curr. Biol. 17, 556-561.

Grimm, C., Gaytan de Ayala Alonso, A., Rybin, V., Steuerwald, U., Ly-Hartig, N., Fischle, W., Müller, J., and Müller, C.W. (2007). Structural and functional analyses of methyl-lysine binding by the malignant brain tumour repeat protein Sex comb on midleg. EMBO Rep. 8, 1031-1037.

Guccione, E., Bassi, C., Casadio, F., Martinato, F., Cesaroni, M., Schuchlautz, H., Luscher, B., and Amati, B. (2007). Methylation of histone H3R2 by PRMT6 and H3K4 by an MLL complex are mutually exclusive. Nature 449, 933-937. Hoffmans, R., and Basler, K. (2004). Identification and in vivo role of the Armadillo-Legless interaction. Development 131, 4393-4400.

Hoffmans, R., Städeli, R., and Basler, K. (2005). Pygopus and legless provide essential transcriptional coactivator functions to armadillo/ $\beta$-catenin. Curr. Biol. 15, 1207-1211.

Jenuwein, T., and Allis, C.D. (2001). Translating the histone code. Science 293 1074-1080.

Kirmizis, A., Santos-Rosa, H., Penkett, C.J., Singer, M.A., Vermeulen, M., Mann, M., Bahler, J., Green, R.D., and Kouzarides, T. (2007). Arginine methylation at histone H3R2 controls deposition of H3K4 trimethylation. Nature 449, 928-932.

Kouzarides, T. (2007). Chromatin modifications and their function. Cell 128, 693-705.

Kramps, T., Peter, O., Brunner, E., Nellen, D., Froesch, B., Chatterjee, S., Murone, M., Züllig, S., and Basler, K. (2002). Wnt/wingless signaling requires BCL9/legless-mediated recruitment of pygopus to the nuclear $\beta$-cateninTCF complex. Cell 109, 47-60.

Lake, B.B., and Kao, K.R. (2003). Pygopus is required for embryonic brain patterning in Xenopus. Dev. Biol. 261, 132-148.

Lan, F., Collins, R.E., De Cegli, R., Alpatov, R., Horton, J.R., Shi, X., Gozani, O., Cheng, X., and Shi, Y. (2007). Recognition of unmethylated histone H3 lysine 4 links BHC80 to LSD1-mediated gene repression. Nature 448, 718-722.

Li, H., llin, S., Wang, W., Duncan, E.M., Wysocka, J., Allis, C.D., and Patel, D.J. (2006). Molecular basis for site-specific read-out of histone H3K4me3 by the BPTF PHD finger of NURF. Nature 442, 91-95.

Li, B., Rheaume, C., Teng, A., Bilanchone, V., Munguia, J.E., Hu, M., Jessen, S., Piccolo, S., Waterman, M.L., and Dai, X. (2007a). Developmental phenotypes and reduced Wnt signaling in mice deficient for pygopus 2. Genesis 45, 318-325.

Li, H., Fischle, W., Wang, W., Duncan, E.M., Liang, L., Murakami-Ishibe, S., Allis, C.D., and Patel, D.J. (2007b). Structural basis for lower lysine methylation 
state-specific readout by MBT repeats of L3MBTL1 and an engineered PHD finger. Mol. Cell 28, 677-691.

McCoy, A.J., Grosse-Kunstleve, R.W., Storoni, L.C., and Read, R.J. (2005). Likelihood-enhanced fast translation functions. Acta Crystallogr. D Biol. Crystallogr. 61, 458-464.

Nakamura, Y., Umehara, T., Hamana, H., Hayashizaki, Y., Inoue, M., Kigawa, T., Shirouzu, M., Terada, T., Tanaka, A., Padmanabhan, B., and Yokoyama, S. (2007). Crystal structure analysis of the PHD domain of the transcription co-activator Pygopus. J. Mol. Biol. 370, 80-92.

Parker, D.S., Jemison, J., and Cadigan, K.M. (2002). Pygopus, a nuclear PHDfinger protein required for Wingless signaling in Drosophila. Development 129, 2565-2576.

Pena, P.V., Davrazou, F., Shi, X., Walter, K.L., Verkhusha, V.V., Gozani, O., Zhao, R., and Kutateladze, T.G. (2006). Molecular mechanism of histone H3K4me3 recognition by plant homeodomain of ING2. Nature 442, 100-103.

Polakis, P. (2000). Wnt signaling and cancer. Genes Dev. 14, 1837-1851.

Ramon-Maiques, S., Kuo, A.J., Carney, D., Matthews, A.G., Oettinger, M.A., Gozani, O., and Yang, W. (2007). The plant homeodomain finger of RAG2 recognizes histone $\mathrm{H} 3$ methylated at both lysine-4 and arginine-2. Proc. Natl. Acad. Sci. USA 104, 18993-18998.

Sampietro, J., Dahlberg, C.L., Cho, U.S., Hinds, T.R., Kimelman, D., and Xu, W. (2006). Crystal structure of a $\beta$-catenin/BCL9/Tcf4 complex. Mol. Cell 24, 293-300.

Schwab, K.R., Patterson, L.T., Hartman, H.A., Song, N., Lang, R.A., Lin, X., and Potter, S.S. (2007). Pygo1 and Pygo2 roles in Wnt signaling in mammalian kidney development. BMC Biol. 5, 15.

Shi, X., Hong, T., Walter, K.L., Ewalt, M., Michishita, E., Hung, T., Carney, D., Pena, P., Lan, F., Kaadige, M.R., et al. (2006). ING2 PHD domain links histone H3 lysine 4 methylation to active gene repression. Nature 442, 96-99.

Shi, X., Kachirskaia, I., Walter, K.L., Kuo, J.H., Lake, A., Davrazou, F., Chan, S.M., Martin, D.G., Fingerman, I.M., Briggs, S.D., et al. (2007). Proteomewide analysis in Saccharomyces cerevisiae identifies several PHD fingers as novel direct and selective binding modules of histone $\mathrm{H} 3$ methylated at either lysine 4 or lysine 36. J. Biol. Chem. 282, 2450-2455.
Sierra, J., Yoshida, T., Joazeiro, C.A., and Jones, K.A. (2006). The APC tumor suppressor counteracts $\beta$-catenin activation and $\mathrm{H} 3 \mathrm{~K} 4$ methylation at Wnt target genes. Genes Dev. 20, 586-600.

Song, N., Schwab, K.R., Patterson, L.T., Yamaguchi, T., Lin, X., Potter, S.S., and Lang, R.A. (2007). pygopus 2 has a crucial, Wnt pathway-independent function in lens induction. Development 134, 1873-1885.

Städeli, R., and Basler, K. (2005). Dissecting nuclear Wingless signalling: recruitment of the transcriptional co-activator Pygopus by a chain of adaptor proteins. Mech. Dev. 122, 1171-1182.

Stock, D., Perisic, O., and Löwe, J. (2005). Robotic nanolitre protein crystallisation at the MRC Laboratory of Molecular Biology. Prog. Biophys. Mol. Biol. 88, 311-327.

Taverna, S.D., llin, S., Rogers, R.S., Tanny, J.C., Lavender, H., Li, H., Baker, L., Boyle, J., Blair, L.P., Chait, B.T., et al. (2006). Yng1 PHD finger binding to H3 trimethylated at K4 promotes NuA3 HAT activity at K14 of $\mathrm{H} 3$ and transcription at a subset of targeted ORFs. Mol. Cell 24, 785-796.

Taverna, S.D., Li, H., Ruthenburg, A.J., Allis, C.D., and Patel, D.J. (2007). How chromatin-binding modules interpret histone modifications: lessons from professional pocket pickers. Nat. Struct. Mol. Biol. 14, 1025-1040.

Thompson, B.J. (2004). A complex of Armadillo, Legless, and Pygopus coactivates dTCF to activate wingless target genes. Curr. Biol. 14, 458-466.

Thompson, B., Townsley, F., Rosin-Arbesfeld, R., Musisi, H., and Bienz, M. (2002). A new nuclear component of the Wnt signalling pathway. Nat. Cell Biol. 4, 367-373.

Townsley, F.M., Cliffe, A., and Bienz, M. (2004a). Pygopus and Legless target Armadillo/ $\beta$-catenin to the nucleus to enable its transcriptional co-activator function. Nat. Cell Biol. 6, 626-633.

Townsley, F.M., Thompson, B., and Bienz, M. (2004b). Pygopus residues required for its binding to Legless are critical for transcription and development. J. Biol. Chem. 279, 5177-5183.

Willert, K., and Jones, K.A. (2006). Wnt signaling: is the party in the nucleus? Genes Dev. 20, 1394-1404.

Wysocka, J., Swigut, T., Xiao, H., Milne, T.A., Kwon, S.Y., Landry, J., Kauer, M., Tackett, A.J., Chait, B.T., Badenhorst, P., et al. (2006). A PHD finger of NURF couples histone $\mathrm{H} 3$ lysine 4 trimethylation with chromatin remodelling Nature 442, 86-90. 